\title{
Terapéutica de la encefalopatía posterior reversible: una revisión sistemática
}

\section{Therapeutics of posterior reversible encephalopathy: a systematic review}

\author{
Karina Rivarola ${ }^{1}{ }^{1}$, Ignacio Ortiz ${ }^{1}$
}

${ }^{1}$ Universidad Nacional de Asunción, Facultad de Ciencias Médicas, San Lorenzo, Paraguay.

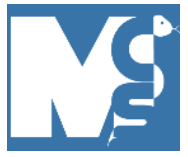

Recibido: 01/02/2021

Revisado: 24/03/2021

Aceptado:30/04/2021

\section{Autor correspondiente}

Karina Rivarola

Universidad Nacional de Asunción,

San Lorenzo, Paraguay

krivarola@med.una.py

\section{Conflictos de interés}

Los autores declaran no poseer conflictos de interés.

\section{Fuente de financiación}

Los autores no recibieron apoyo financiero para la investigación, autoría y/o publicación de este artículo.

Este artículo es publicado bajo una licencia de Creative Commons Reconocimiento 4.0 Internacional.

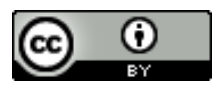

\section{RESUMEN}

Introducción: Síndrome de encefalopatía posterior reversible [PRES (también conocido como síndrome de leucoencefalopatía posterior reversible)] se presenta con síntomas de inicio rápido como cefalea, convulsiones, alteración de la conciencia y alteración visual. A menudo, pero de ninguna manera siempre, se asocia con la hipertensión aguda. Metodología: Estudio de tipo revisión sistemática de la literatura. Se realizó una revisión sistemática de la literatura en las bases de datos PubMed/MEDLINE, LILACS y CICCO utilizando la sintaxis "Posterior reversible encephalopathy syndrome AND treatment". Los límites de búsqueda fueron publicaciones hechas en los últimos cinco años (2013-2020). Resultados: Se evaluaron 235 textos completos de los cuales finalmente se seleccionaron seis, esto teniendo en cuenta que los demás artículos no mencionaban de forma explícita el tratamiento, o eran redundantes con respecto a otros ya incluidos, teniendo en cuenta que el PRES tiene un manejo sintomático más que un tratamiento específico. Discusión: Síndrome de Encefalopatía Posterior Reversible (PRES) no tiene un tratamiento específico, sino más bien tiene un tratamiento sintomático lo que hace fundamental conocer las causas subyacentes al PRES ya que esa es la terapéutica a seguir, brindar el mejor tratamiento para lo que está causando este síndrome, de allí la importancia de conocer a fondo la etiología.

Palabras clave: Encefalopatías; Síndrome de Leucoencefalopaía Posterior; Terapéutica.

\section{ABSTRACT}

Introduction: Posterior reversible encephalopathy syndrome [PRES (also known as posterior reversible leukoencephalopathy syndrome)] presents with rapid-onset symptoms such as headache, seizures, altered consciousness, and visual impairment. Often, but by no means always, it is associated with acute hypertension. Methods: Systematic literature review type study. A systematic review of the literature was carried out in the PubMed / MEDLINE, LILACS and CICCO databases using the syntax "Posterior reversible encephalopathy syndrome AND treatment". The search limits were publications made in the last five years (2013-2020). Results: 235 full texts were evaluated, of which six were finally selected, taking into account that the other articles did not explicitly mention the treatment, or were redundant with respect to others already included, taking into account that the PRES has symptomatic rather than specific treatment. Discussion: Posterior Reversible Encephalopathy Syndrome (PRES) does not have a specific treatment, but rather has a symptomatic treatment, which makes it essential to know the underlying causes of PRES since that is the therapy to follow, to provide the best treatment for what is causing this syndrome, hence the importance of fully understanding the etiology.

Keywords: Encephalopathies; Posterior Leukoencephalopaia Syndrome; Therapy. 


\section{INTRODUCCIÓN}

Síndrome de encefalopatía posterior reversible [PRES (también conocido como síndrome de leucoencefalopatía posterior reversible)] se presenta con síntomas de inicio rápido como cefalea, convulsiones, alteración de la conciencia y alteración visual. A menudo, pero de ninguna manera siempre, se asocia con la hipertensión aguda. Si se reconoce y se trata rápidamente, el síndrome clínico generalmente desaparece en una semana y los cambios observados en la resonancia magnética (RM) se resuelven entre días y semanas (1).

La gravedad de los síntomas clínicos varía. Por ejemplo, la alteración visual puede manifestarse como visión borrosa, hemianopsia homónima o incluso ceguera cortical (2). Los pacientes pueden estar levemente confundidos o agitados, pero pueden volverse comatosos (2). Otros síntomas menos frecuentes son las náuseas, los vómitos y los déficits en el tallo cerebral $(2,3)$. Las convulsiones y el estado epiléptico son comunes, y el estado epiléptico no convulsivo puede ser más frecuente que el estado epiléptico generalizado (4).

Se debe sospechar el estado no convulsivo en pacientes con estados prolongados de conciencia alterada y se puede confundir con el estado postictal. Los signos de convulsiones no convulsivas incluyen movimientos estereotípicos como mirar fijamente, parpadear o girar la cabeza. El estado postictal dura varias horas, pero el PRES y el estado no convulsivo pueden persistir durante varios días y confundirse con psicosis, intoxicación por drogas o estados psicógenos (5).

La enfermedad renal crónica y la lesión renal aguda están ambas comúnmente presentes en pacientes con PRES, y el PRES está fuertemente asociada con condiciones que coexisten en pacientes con enfermedad renal, como hipertensión, enfermedades vasculares y autoinmunes, exposición a fármacos inmunosupresores, y trasplante de órganos. Por lo tanto, es importante considerar PRES en el diagnóstico diferencial de pacientes con enfermedad renal y síntomas neurológicos rápidamente progresivos. El síndrome de encefalopatía posterior reversible es un trastorno cada vez más reconocido, con un amplio espectro clínico de síntomas y factores desencadenantes, $y$, sin embargo, sigue siendo poco conocido (1).
Como hemos visto, esta patología es rica en semiología y hallazgos imagenológicos, además pocas veces se piensa en una encefalopatía posterior reversible como un primer diagnóstico, sino más bien, ser hace luego de descargar otros, así el objetivo principal de esta investigación es determinar el perfil clínico epidemiológico de los pacientes estudiados que tiene diagnóstico de PRES.

\section{METODOLOGÍA}

Estudio de tipo revisión sistemática de la literatura. Se realizó una revisión sistemática de la literatura en las bases de datos PubMed/MEDLINE, LILACS y CICCO utilizando la sintaxis "Posterior reversible encephalopathy syndrome AND treatment". Los límites de búsqueda fueron publicaciones hechas en los últimos cinco años (2013-2020). Los tipos de estudio incorporados fueron: revisión sistemática, reporte de casos, ensayo clínico, estudio comparativo, estudios de evaluación, estudios multicéntricos, estudios de casos y controles, estudios observacionales, estudios de cohorte y metaanálisis.

Se incluyó a todos los artículos que fueron publicados en revistas científicas arbitradas y que estén disponibles a texto completo en idioma inglés o español. Posteriormente de clasificar los resultados se procedió a recuperar los artículos en formato digital para realizar una lectura crítica de la evidencia teniendo en cuenta los criterios de inclusión, y así cumplir con el objetivo de la investigación.

La presente investigación no presenta conflictos éticos ya que no involucra a pacientes. Se respetarán los criterios de búsqueda y se citarán las fuentes que fueron incluidas en la investigación.

\section{RESULTADOS}

\section{Descripción de los resultados}

Se realizó una revisión sistemática de la literatura con el fin de describir los tratamientos más actuales para el PRES de acuerdo a las últimas publicaciones médicas en las principales bases de datos. Se evaluaron 235 textos completos de los cuales finalmente se seleccionaron seis, esto teniendo en cuenta que los demás artículos no mencionaban de forma explícita el tratamiento, o eran redundantes con respecto a otros ya incluidos, teniendo en cuenta que el PRES tiene un manejo sintomático más que un tratamiento específico. 
FIGURA 1. ALGORITMO DE LA REVISIÓN SISTEMÁTICA

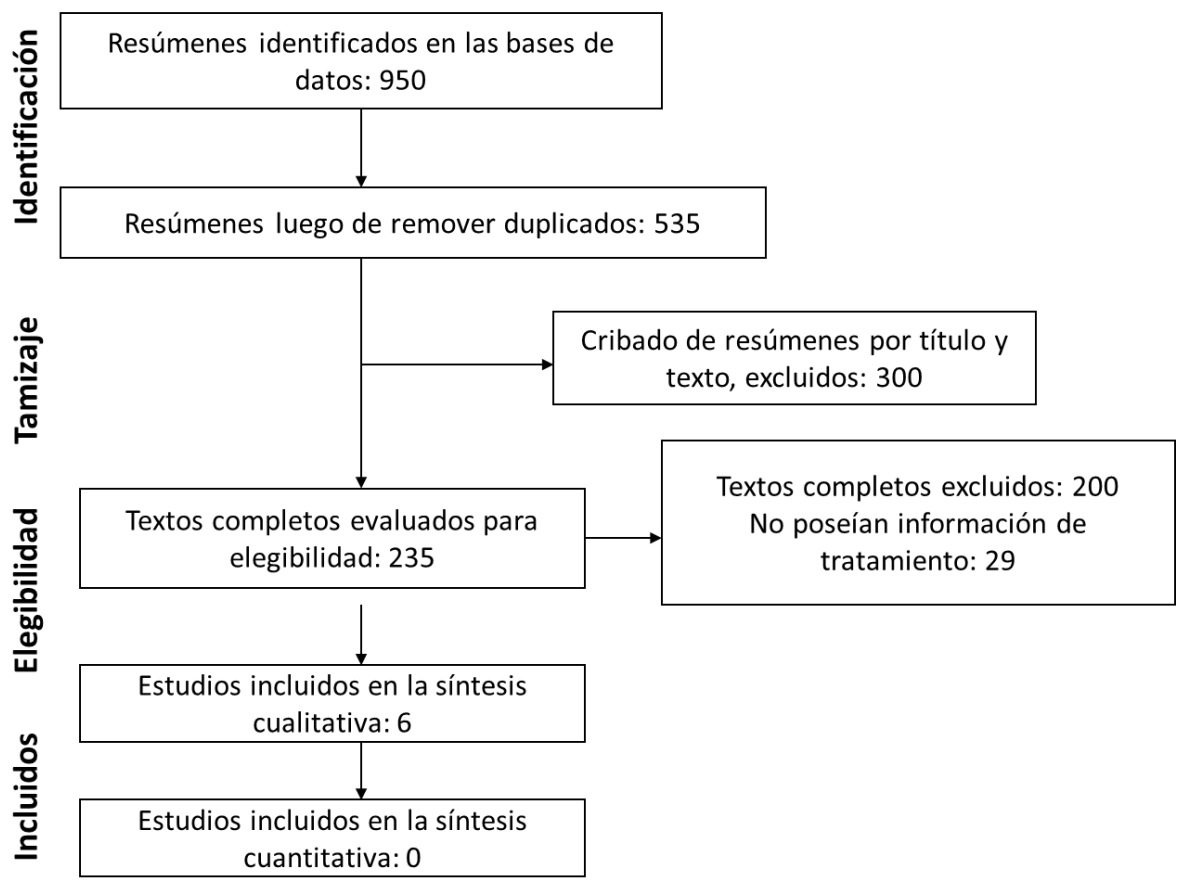

\section{Síntesis cualitativa de los hallazgos}

El tratamiento de la PRES es sintomático, ya que actualmente no se dispone de una estrategia terapéutica específica. El manejo de la enfermedad o patología subyacente que conduce al desarrollo de PRES es de gran importancia. El síndrome PRES debe tratarse de manera temprana, agresiva y durante un tiempo suficientemente prolongado para prevenir las recaídas. Un diagnóstico precoz es el primer paso importante para lograr un tratamiento eficaz.

El tratamiento de los episodios de hipertensión y el mantenimiento de la presión arterial normal es un componente esencial del tratamiento con PRES. Sin embargo, no hay pruebas, basadas en estudios prospectivos controlados, de que el control estricto de la presión arterial limite la lesión neurológica, o resulte en una regresión de los hallazgos clínicos o de imagen. La elección de los fármacos antihipertensivos se basa en recomendaciones generales para el manejo de crisis hipertensivas o emergencias hipertensivas. Se recomienda una reducción de los niveles de presión arterial en un $25 \%$ de los valores de referencia. Al igual que con otras afecciones, deben evitarse las fluctuaciones de la presión arterial y debe considerarse la administración continua de fármacos antihipertensivos bajo control hemodinámico (6).

La estrategia de tratamiento relaciona medidas generales para la corrección de la causa subyacente de PRES. Un diagnóstico etiológico temprano permite la corrección inmediata de la causa de PRES. Los pacientes pueden necesitar control de la presión arterial, retirada de quimioterapia contra el cáncer o agentes inmunosupresores, cesárea, diálisis u otras intervenciones. La corrección inmediata de la causa es esencial para disminuir el riesgo de isquemia o hemorragia $\mathrm{y}$, por lo tanto, para prevenir la discapacidad permanente o la muerte.

En pacientes con convulsiones, se recomienda la terapia anticonvulsiva incluso después de una sola convulsión. Se recomienda la conversión de la terapia anticonvulsiva a fármacos inductores de enzimas microsomales no hepáticos tan pronto como la situación clínica del paciente se estabilice. Se recomienda suspender el tratamiento anticonvulsivo después de 3-6 meses en casos no complicados. También se recomienda un régimen anticonvulsivo durante al menos 12 meses después del siguiente episodio convulsivo en pacientes con hallazgos anormales en el electroencefalograma o la resonancia magnética craneal y las convulsiones recurrentes. Aún no se ha logrado un consenso sobre qué pacientes deberían recibir fármacos antiepilépticos para las convulsiones después de PRES (7-10).

El control de volumen a menudo es un factor importante en el manejo de la presión arterial, particularmente en casos de PRES que ocurren en pacientes bajo diálisis crónica; el tratamiento dialítico podría ayudar a restablecer la presión arterial adecuada en algunos casos. Si se diagnostica eclampsia o preeclampsia, se indica la suplementación con sulfato de magnesio; podría ser necesaria un parto prematuro (11). En el caso de hipertensión relacionada con feocromocitoma, el tratamiento antihipertensivo debe considerar nitroprusiato y fentolamina; El sulfato de 
magnesio también parece tener un efecto específico para el feocromocitoma. Se ha descrito la agravación de la PRES con nitroglicerina $y$, por lo tanto, se debe evitar este medicamento. Los agentes antiepilépticos preferidos para controlar las convulsiones son agentes intravenosos que pueden cargarse rápidamente, como las benzodiazepinas intravenosas; Los anticonvulsivos DISCUSIÓN

Como se ha visto el Síndrome de Encefalopatía Posterior Reversible (PRES) no tiene un tratamiento específico, sino más bien tiene un tratamiento sintomático lo que hace fundamental conocer las causas subyacentes al PRES ya que esa es la terapéutica a seguir, brindar el mejor tratamiento para lo que está causando este síndrome, de allí la importancia de conocer a fondo la etiología.

Así como es importante conocer la etiología, también es fundamental conocer las características clínicas y las características imagenológicas de este síndrome con el fin de un diagnóstico precoz y tratamiento oportuno. Por lo cual se hará una breve mención a los aspectos clínicos e imagenológicos del PRES.

Los principales signos y síntomas presentes en este tipo de pacientes son: Encefalopatía (50-80 \%), convulsiones (60-75\%), cefalea (50\%), trastornos visuales (33\%), déficit neurológico focal (10-15\%), estado epiléptico (5-15\%) (12-18). En cuanto al diagnóstico, los síntomas neurológicos agudos y subagudos en el contexto clínico apropiado (es decir, en presencia de hipertensión pronunciada, fluctuaciones de la presión arterial, inmunodepresión, trastornos autoinmunes, insuficiencia renal, preeclampsia o eclampsia) sugieren PRES. Los síntomas y signos de PRES no son específicos y se pueden observar en muchos otros trastornos neurológicos. En el contexto agudo, los clínicos pueden tener dificultades para averiguar si la presión arterial elevada es primaria o secundaria, ya que otras causas (intoxicación por drogas, abstinencia de alcohol, accidente cerebrovascular agudo) pueden dar lugar a pronunciados síntomas fisiológicos de hipertensión. No existen pautas para dirigir esta evaluación; por lo tanto, el juicio clínico es crucial. El diagnóstico diferencial de PRES incluye muchas posibilidades. Los síntomas y signos son inespecíficos de forma aislada, por lo que requieren imágenes del cerebro con la intención primaria de excluir diagnósticos alternativos. Sin embargo, el diagnóstico de PRES no es principalmente radiológico, el contexto clínico y el juicio del clínico son cruciales para hacer el diagnóstico correcto (12).

En cuanto a las imágenes, las imágenes cerebrales son útiles para excluir diagnósticos alternativos, pero de segunda línea podrían ser fenobarbital y fenitoína. En pacientes con actividad convulsiva continua a pesar de las benzodiacepinas intravenosas y fenobarbital / fenitoína, debe considerarse la infusión de dosis tituladas de midazolam, propofol o tiopental hasta la remisión de la actividad convulsiva clínica (11).

también suelen confirmar un diagnóstico de PRES. Sin embargo, incluso cuando la RM cerebral muestra regiones de señal T2 anormal el diagnóstico diferencial todavía podría ser extenso. Aunque el edema vasogénico se puede visualizar mediante una TC sin contraste en algunos pacientes, la RM cerebral es mucho más sensible. Las imágenes cerebrales generalmente revelan edema vasogénico en las regiones parieto-occipitales de ambos hemisferios cerebrales, la sustancia blanca subcortical siempre está afectada y la corteza también suele estar involucrada. El edema suele ser asimétrico, pero casi siempre bilateral (12).

En cuanto al manejo clínico actual se menciona en la sección de resultados que básicamente se trata como si fuera una crisis hipertensiva aguda con la salvedad de que se debe identificar la causa subyacente y brincar un tratamiento específico para dicha causa para que así el síndrome se resuelva. Se puede concluir que la literatura sobre el manejo terapéutico del PRES no es abundante ya que depende de la etiología para brindar tratamiento, siendo mucho más importante un buen diagnóstico clínico y con los métodos auxiliares de diagnóstico adecuados.

\section{CONTRIBUCIÓN DE LOS AUTORES}

KR: Concepción y diseño del estudio, procesamiento y análisis de los datos, redacción del manuscrito, revisión de la literatura, aprobación de la versión final del documento. 10: Concepción y diseño del estudio, procesamiento y análisis de los datos, revisión crítica del manuscrito, revisión de la literatura, aprobación de la versión final del manuscrito.

\section{REFERENCIAS}

1. Hobson EV, Craven I, Blank SC. Posterior Reversible Encephalopathy Syndrome: A Truly Treatable Neurologic Illness. Perit Dial Int J Int Soc Perit Dial. 2012;32(6):590-594. https://dx.doi.org/10.3747\%2Fpdi.2012.00152

2. Roth C, Ferbert A. The posterior reversible encephalopathy syndrome: what's certain, what's new? Pract Neurol. 2011;11(3):136-144. https://doi.org/10.1136/practneurol2011-000010

3. Hinchey J, Chaves C, Appignani B, Breen J, Pao L, Wang A, et al. A reversible posterior leukoencephalopathy syndrome. $N$ Engl J Med. 1996;334(8):494-500. https://doi.org/10.1056/neim199602223340803

4. Kozak OS, Wijdicks EFM, Manno EM, Miley JT, Rabinstein AA. Status epilepticus as initial manifestation of posterior 
reversible encephalopathy syndrome. Neurology. 2007;69(9):894-897.

https://doi.org/10.1212/01.wnl.0000269780.45472.16

5. Kaplan PW. No, some types of nonconvulsive status epilepticus cause little permanent neurologic sequelae (or: "the cure may be worse than the disease"). Neurophysiol Clin Clin Neurophysiol. 2000;30(6):377-382. https://doi.org/10.1016/s0987-7053(00)00238-0

6. Pak KJ, Hu T, Fee C, Wang R, Smith M, Bazzano LA. Acute Hypertension: A Systematic Review and Appraisal of Guidelines. Ochsner J. 2014;14(4):655-663. URL.

7. Legriel S, Pico F, Azoulay E. Understanding Posterior Reversible Encephalopathy Syndrome. En: Vincent J-L, editor. Annual Update in Intensive Care and Emergency Medicine 2011. Berlin, Heidelberg: Springer Berlin Heidelberg; 2011. p. 631-53.

8. Kim SJ, Im SA, Lee JW, Chung NG, Cho B, Kim HK, et al. Predisposing factors of posterior reversible encephalopathy syndrome in acute childhood leukemia. Pediatr Neurol. 2012;47(6):436-442. https://doi.org/10.1016/j.pediatrneurol.2012.07.011

9. de Laat $P$, Te Winkel ML, Devos AS, Catsman-Berrevoets CE, Pieters R, van den Heuvel-Eibrink MM. Posterior reversible encephalopathy syndrome in childhood cancer. Ann Oncol Off J Eur Soc Med Oncol. 2011;22(2):472-478. https://doi.org/10.1093/annonc/mdq382

10. Siebert E, Spors B, Bohner G, Endres M, Liman TG. Posterior reversible encephalopathy syndrome in children: radiological and clinical findings - a retrospective analysis of a German tertiary care center. Eur J Paediatr Neurol EJPN Off J Eur Paediatr Neurol Soc. 2013;17(2):169-175. https://doi.org/10.1016/i.ejpn.2012.08.003

11. Granata G, Greco A, lannella G, Granata M, Manno A, Savastano $E$, et al. Posterior reversible encephalopathy syndrome-Insight into pathogenesis, clinical variants and treatment approaches. Autoimmun Rev. 2015;14(9):830836. https://doi.org/10.1016/j.autrev.2015.05.006

12. Fugate JE, Rabinstein AA. Posterior reversible encephalopathy syndrome: clinical and radiological manifestations, pathophysiology, and outstanding questions. Lancet Neurol. 2015;14(9):914-925. https://doi.org/10.1016/s1474-4422(15)00111-8

13. Burnett MM, Hess CP, Roberts JP, Bass NM, Douglas VC, Josephson SA. Presentation of reversible posterior leukoencephalopathy syndrome in patients on calcineurin inhibitors. Clin Neurol Neurosurg. 2010;112(10):886-891. https://doi.org/10.1016/i.clineuro.2010.07.023

14. Liman TG, Bohner G, Heuschmann PU, Endres M, Siebert $E$. The clinical and radiological spectrum of posterior reversible encephalopathy syndrome: the retrospective Berlin PRES study. J Neurol. 2012;259(1):155-164. https://doi.org/10.1007/s00415-011-6152-4

15. Li $Y$, Gor D, Walicki D, Jenny D, Jones $D$, Barbour $P$, et al. Spectrum and potential pathogenesis of reversible posterior leukoencephalopathy syndrome. J Stroke Cerebrovasc Dis Off J Natl Stroke Assoc. 2012;21(8):873882.

https://doi.org/10.1016/j.jstrokecerebrovasdis.2011.05.01 0

16. Cruz RJ, DiMartini A, Akhavanheidari M, lacovoni N, Boardman JF, Donaldson J, et al. Posterior reversible encephalopathy syndrome in liver transplant patients: clinical presentation, risk factors and initial management. Am J Transplant Off J Am Soc Transplant Am Soc Transpl Surg. 2012;12(8):2228-2236. https://doi.org/10.1111/j.1600-6143.2012.04048.x
17. Legriel S, Schraub O, Azoulay E, Hantson P, Magalhaes E, Coquet I, et al. Determinants of recovery from severe posterior reversible encephalopathy syndrome. PloS One. 2012;7(9):e44534 https://doi.org/10.1371/journal.pone.0044534

18. Brewer J, Owens MY, Wallace K, Reeves AA, Morris R, Khan $M$, et al. Posterior reversible encephalopathy syndrome in 46 of 47 patients with eclampsia. Am J Obstet Gynecol. 2013;208(6):468.e1-6. https://doi.org/10.1016/j.ajog.2013.02.015 